\title{
Pediatric anesthesia: current status and future directions
}

\author{
Norifumi Kuratani $^{1} \cdot$ Yuichi Kanmura ${ }^{2}$
}

Received: 26 October 2015 / Accepted: 2 November 2015 / Published online: 25 November 2015

(C) Japanese Society of Anesthesiologists 2015

As part of the 62nd Annual Meeting of the Japanese Society of Anesthesiologists, the 10th Journal of Anesthesia (JA) Symposium was held on May 28, 2015 in Kobe, Japan. The JA Symposium has been convened annually since 2006. The goals of the symposium are to facilitate cutting-edge discussion at the forefront of anesthesiology and to promote JA among the anesthesia community around the world.

This year's JA symposium focused on pediatric anesthesia, and the title of the symposium was "Pediatric Anesthesia: Current Status and Future Directions." Five distinguished speakers gathered to discuss their respective areas of expertise. Two moderators, Dr. Kanmura and Dr. Kuratani, facilitated the discussions.

The first speaker, Dr. Hiromi Kako of Nationwide Children's Hospital, Columbus, OH, USA, discussed topics related to the use of the cuffed endotracheal tube (ETT) in the pediatric population. The use of the cuffed ETT in small children and infants has been a controversial issue in the pediatric anesthesia community. The advantages of the cuffed ETT are obvious, yet safety concerns associated with it are yet to be completely addressed. Newly invented cuffed ETTs with thin polyurethane microcuffs are said to overcome some of the disadvantages related to cuff design in the previously available polyvinyl chloride (PVC) cuffs, and the new generation of cuffed ETTs may be a game changer in pediatric airway management. Dr. Kako,

Norifumi Kuratani

nori-kuratani@umin.ac.jp

1 Department of Anesthesia, Saitama Children's Medical Center, Saitama, Japan

2 Department of Anesthesiology and Intensive Care, Kagoshima University Hospital, Kagoshima, Japan however, emphasized that, even when the new ETT is used, close attention must be paid to maintaining cuff pressure within the appropriate range to avoiding postoperative airway complications. In particular, it has been shown that cuff pressure is not always stable throughout anesthesia. As cuff pressure is affected by various factors, including patient positioning, continuous monitoring and adjustment of cuff pressure are strongly advocated [1].

Dr. Soichiro Obara of Saitama Children's Medical Center, Japan, covered issues related to adverse events associated with the management of the pediatric airway outside the operating room (OR), especially in the pediatric emergency department and the pediatric intensive care unit and during sedation of infants and children, including the ramifications of the latest literature. The question of which strategies are most effective at helping non-anesthesia trainees to achieve proficiency in pediatric airway management is the central issue here. He presented the possible solutions, including simulation training and the formation of a formal multidisciplinary team of airway specialists known as the Difficult Airway Service [2], and noted that both of these solutions would be most effective if directed by anesthesiologists. Although it remains somewhat unclear whether the involvement of anesthesiologists contributes to safety in the management of the pediatric airway outside the OR, Dr. Obara stressed the importance of anesthesiologists' efforts to reach out and get involved in the issue through close coordination and collaboration with other departments.

Dr. Yuji Kitamura of the Montréal Children's Hospital, Montréal, Canada, discussed perioperative anesthetic management in tonsillectomy and adenoidectomy (T\&A) for children with severe obstructive sleep apnea (OSA). In North America, where T\&A is often conducted on an ambulatory basis, out-of-hospital deaths in the early days 
following T\&A have recently been reported and are often attributed to apnea after the return home. Perioperative anesthetic management during this procedure is based on clinical research done by Dr. Karen Brown at Montréal Children's Hospital [3]. Dr. Kitamura especially emphasized the importance of considering the severity of OSA to ensure safer management. It is difficult to assess the severity of OSA through questionnaires alone; polysomnography is necessary for a complete assessment. The McGill Oximetry Score is available as an alternative to polysomnography.

Dr. Yasushi Satoh of the National Defense Medical College, Tokorozawa, Saitama, Japan, discussed the neurotoxic effects of anesthetics on the developing nervous system. The critical period is a distinct time window during the neonatal stage when animals display elevated sensitivity to certain environmental stimuli, and particular experiences can have profound and long-lasting effects on behaviors. Increasing evidence suggests that neonatal exposure to general anesthetics during the critical period induces significantly increased brain apoptosis with subsequent behavioral deficits later in adulthood, although the pathogenic mechanism is largely unknown. Dr. Satoh showed that extracellular signal-regulated protein kinases (ERKs) play important roles in the proper formation of neural circuits during the critical period. Transient blockade of ERK phosphorylation at postnatal day 6 (P6) by intraperitoneal injection of the blood-brain barrier-penetrating MEK inhibitor $\alpha$-[amino[(4-aminophenyl)thio]methylene]-2(trifluoromethyl)benzeneacetonitrile (SL327) caused a significant increase in apoptosis in the forebrain. This increase in apoptosis induced long-term deleterious effects on brain function that emerged later in adulthood, resulting in social deficits, impaired memory, and reduced long-term potentiation (LTP). Conversely, blockade of ERK phosphorylation at postnatal day 14 (P14) no longer induced apoptosis or behavioral deficits. These results indicate that phosphorylation of ERKs during the critical period is absolutely required for normal development of the central nervous system. This study provides novel insight into the mechanistic basis for anesthetic-induced toxicity in the developing brain [4].
The final presentation was by Dr. Akihiro Kanaya of Tohoku University Hospital, Miyagi, Japan, who spoke about emergence agitation (EA) in pediatric anesthesia. EA immediately after extubation is a difficult problem in routine pediatric anesthesia. Dr. Kanaya emphasized that no single factor can explain why excitatory emergence occurs in pediatric patients. He pointed out, however, that the choice of anesthetics can be a highly significant modifiable factor in EA; for example, sevoflurane maintenance is associated with a higher incidence of EA compared to intravenous anesthesia using propofol [5]. Dr. Kanaya also noted that the use of sedatives to prevent EA might result in unexpected delays in anesthesia recovery and might cause airway obstruction during the recovery phase [6].

Unfortunately, time ran short during the event, and the wrap-up discussion was incomplete. As the speakers were strongly encouraged to submit review articles to JA, however, we expect to continue our vigorous discussion of the latest research on these topics in our journal.

\section{References}

1. Kako H, Krishna SG, Ramesh AS, Merz MN, Elmaraghy C, Grischkan J, Jatana KR, Ruda J, Tobias JD. The relationship between head and neck position and endotracheal tube intracuff pressure in the pediatric population. Paediatr Anaesth. 2014;24:316-21.

2. Sheeran PW, Walsh BK, Finley AM, Martin AK, Brenski AC. Management of difficult airway patients and the use of a difficult airway registry at a tertiary care pediatric hospital. Paediatr Anaesth. 2014;24:819-24.

3. Raghavendran S, Bagry H, Detheux G, Zhang X, Brouillette RT, Brown KA. An anesthetic management protocol to decrease respiratory complications after adenotonsillectomy in children with severe sleep apnea. Anesth Analg. 2010;110:1093-101.

4. Yufune S, Satoh Y, Takamatsu I, Ohta H, Kobayashi Y, Takaenoki Y, Pagès G, Pouysségur J, Endo S, Kazama T. Transient blockade of ERK phosphorylation in the critical period causes autistic phenotypes as an adult in mice. Sci Rep. 2015;5:10252.

5. Kanaya A, Kuratani N, Satoh D, Kurosawa S. Lower incidence of emergence agitation in children after propofol anesthesia compared with sevoflurane: a meta-analysis of randomized controlled trials. J Anesth. 2014;28:4-11.

6. Kanaya A. Emergence agitation in children: risk factors, prevention, and treatment. J Anesth 2015 (in press). 